\title{
A Case Report of Mucormycosis of Faciotemporal Region Mimicking as Bacterial Necrotizing Fasciitis
}

\author{
Waqar Jeelani, Yousaf Kamal
}

\begin{abstract}
Mucormycosis is an invasive and unyielding fungal disease of immunocompromised patients with a reported increase in incidence over last couple of decades. The close resemblance of clinical features of mucormycosis with that of bacterial necrotizing fasciitis can lead to misdiagnosis and thus delay in appropriate management. The case presented here involves a similar scenario when a middle-aged patient with multiple systemic problems reported to the tertiary care hospital 7 days after facial trauma from a road traffic accident. There was a purple black ulcerating lesion over left midface that was misdiagnosed and treated as a case of bacterial necrotizing fasciitis. Despite extensive debridement under general anesthesia and administration of parentral antibiotics, the patient failed to survive. The results of histopathological examination of the debrided tissue, that became available after the death of the patient showed, growth of broad aseptate hyphae characteristic of mucorales. Late presentation, multiple systemic diseases and missed diagnosis of the condition were the main factors that resulted in the death of the patient within 72 hours of presentation.

Underlying immunocompromised states and late diagnosis of this condition remain the key issues that need to be addressed in developing countries. A high degree of clinical suspicion and aggressive diagnostic measures combined with prompt medical and surgical treatment are essential to improve the outcome.
\end{abstract}

Keywords: Mucormycosis, Necrotizing fasciitis, Immunocompromised, Mucorales, Zygomycosis.

How to cite this article: J eelani W, Kamal Y. A C ase Report of Mucormycosis of Faciotemporal R egion Mimicking as Bacterial Necrotizing Fasciitis. World J Dent 2012;3(4):355-358.

\section{Source of support Nil}

Conflict of interest: None declared

\section{INTRODUCTION}

M ucormycosis, also known as zygomycosis, is an invasive and rapidly progressive fungal disease of immunocompromised population. The incidence of this disease is only 0.7 to 1.2 /million but recent studies showed its increasing incidence over last two decades. ${ }^{1} \mathrm{M}$ ucormycosis can present in different forms including rhinocerebral, pulmonary, cutaneous, gastrointestinal and disseminated. ${ }^{2}$

There is a fair likelihood of this condition to be misdiagnosed as bacterial necrotizing fasciitis based upon its similar clinical features and progressive nature. ${ }^{3}$ The only way to make a definite diagnosis of this disease is by performing a fungal culture and by detecting the characteristic hyphae in different sections of the infected tissues. ${ }^{4}$
The case presented here involved a middle-aged patient with multiple systemic problems having mucormycosis of left facial region secondary to a road side accident. The patient was misdiagnosed and was treated as a case of bacterial necrotizing fasciitis in the absence of timely reports of histopathological examination.

\section{CASE REPORT}

A 53-year-old obese male reported to the emergency room with the chief complaint of an ulcer on left cheek and irritability and drow siness for last 4 days. Patient had history of road traffic accident 7 days back and was taken to a local hospital for treatment. He was discharged from the hospital on oral antibiotics after a stay of 3 days. Despite the use of antibiotics the abrasion on left cheek did not show any signs of healing while the mental condition of the patient started to deteriorate.

The patient was semiconscious and had toxic appearance at the time of presentation. There was a noxious abrasive lesion on left cheek that was purple black in color and was involving whole left midface. The lesion was advancing to the temporal region with edema extending to the vertex of cranium (Fig. 1). There was moderate periorbital swelling and a boney step defect was pal pable at lower orbital margin on left side. All other facial bones were clinically intact and the occlusal relationship between two dental arches was normal (Fig. 2).

A large number of biochemical and radiological investigations were requested (Table 1). Patient was found to be suffering from undiagnosed diabetes mellitus, sinus bradycardia, disseminated intravascular coagulation and thrombocytopenia. M oreover, the CT scan of brain showed intracerebral bleeding in right basal ganglion (Fig. 3). A n emergency debridement of the necrotizing lesion was planned under general anesthesia but patient was declared unfit for surgery due to his debilitating medical condition.

A multidisciplinary approach was implemented to improve the general medical condition of the patient. Three units of platel et concentrates were transfused and platelet count was maintained above $95,000 / \mathrm{mm}^{3}$ The random blood sugar was maintained below $200 \mathrm{mg} / \mathrm{dl}$ by intravenous administration of insulin. The patient was affirmed as a low risk for general anesthesia by the cardiologist while the neurosurgeon recommended conservative management of 
Table 1: A large number of investigations were carried out which showed that patient was suffering from multiple systemic disorders

\begin{tabular}{|c|c|}
\hline Investigations & Results \\
\hline Blood chemistry & $\begin{array}{l}\text { Thrombocytopenia with } \\
\text { platelet count } 72,000 / \mathrm{mm}^{3}\end{array}$ \\
\hline $\begin{array}{l}\text { Fibrin degradation products } \\
\text { (FDP) }\end{array}$ & $\begin{array}{l}\text { Strongly positive (>20 } \mu \mathrm{g} / \mathrm{ml}) \\
\text { suggestive of DIC }\end{array}$ \\
\hline D-Dimer & $>9,000 \mathrm{ng} / \mathrm{ml}$ suggestive of DIC \\
\hline PT/APTT & Within normal limits \\
\hline Anti-HCV, HBsAg & Negative \\
\hline Blood glucose (random) & $\begin{array}{l}\text { Diabetes mellitus with blood } \\
\text { glucose } 384 \mathrm{mg} / \mathrm{dl}\end{array}$ \\
\hline $\begin{array}{l}\text { PA and lateral cephalogram, } \\
\text { Water's view }\end{array}$ & $\begin{array}{l}\text { Fracture of left infraorbital } \\
\text { margin }\end{array}$ \\
\hline CT scan brain & Left basal ganglion bleeding \\
\hline E lectrocardiography (ECG) & Sinus bradycardia \\
\hline Blood $\mathrm{HCO}_{3}$ & $\begin{array}{l}21.6 \mathrm{mE} \mathrm{q} / \mathrm{l} \text { suggestive of } \\
\text { metabolic acidosis }\end{array}$ \\
\hline $\begin{array}{l}\text { Histopathological } \\
\text { examination* }\end{array}$ & $\begin{array}{l}\text { Broad aseptate hyphae } \\
\text { suggestive of mucorales }\end{array}$ \\
\hline Microbiological culture* & No growth of any microbes \\
\hline
\end{tabular}

*Reports were available after death of the patient

Table 2: The drugs administered to the patient

Drugs administered prior to hospitalization:

- Inj. Sulzone (Cefoperazone + Sulbactam) 2 gm IV BID

- Inj. Alpha GPC 250 mg IV BID

- Inj. Insulin SC-sliding scale

Drugs administered presurgically:

- Inj. Cephradine 500 mg IV TID

- Inj. Metronidazole 500 mg IV TID

- Inj. Vancomycin IV 1 gm BID

- Inj. Gentamycin IV $1 \mathrm{gm}$ BID

- Inj. Diclofenac sodium 75 mg IM TID

- Inf. R/L 1,000 mg IV OD

- Inf. 5\% Dextrose +1 amp KCI +12 unit insulin (regular) IV $80 \mathrm{ml} /$ hour

Drugs administered postsurgically:

- Inj. Clindamycin 600 mg IV TDS

- Inj. Ketorolac IM TDS

- Inj. Vancomycin IV 1 gm BID

- Inj. Ceftriaxone $1 \mathrm{gm}$ BID

- Inj. Metronidazole 500 mg IV TID

- Inj. Enoxaparin 40 mg SC OD

- Inj. Omeprazole 40 mg IV OD

Drugs used for resuscitation:

- Inj. Dopamine $6 \mathrm{ug} / \mathrm{kg} / \mathrm{min}$

- Inj. Dobutamine $2 \mathrm{ug} / \mathrm{kg} / \mathrm{min}$

basal ganglion bleeding. Thus, patient was ready to undergo debridement under general anesthesia after a delay of about 24 hours. By this time, the lesion had spread significantly, edema was noticed on the contralateral side of the face and the signs of dyspnea had become obvious. Table 2 summarizes the details of drugs administered to the patient.

Elective tracheostomy was carried out under general anesthesia. The debridement of all the necrotic tissues was

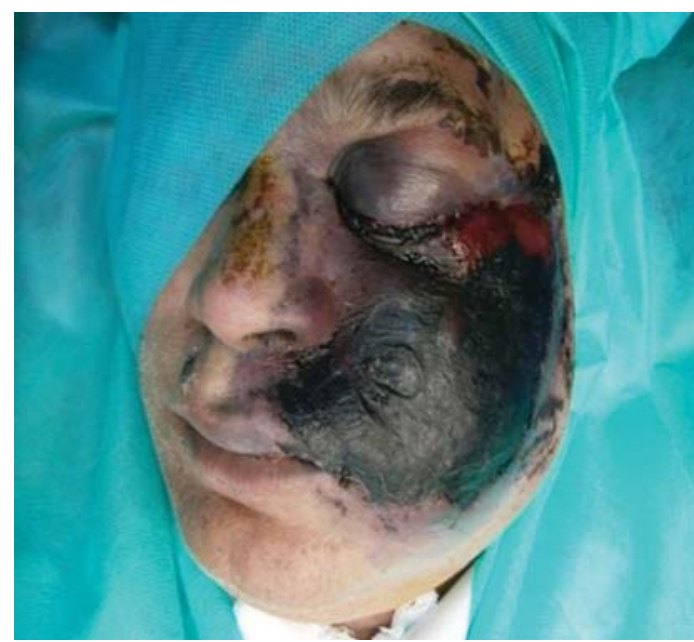

Fig. 1: Rapidly spreading purple black cutaneous lesion of mucormycosis

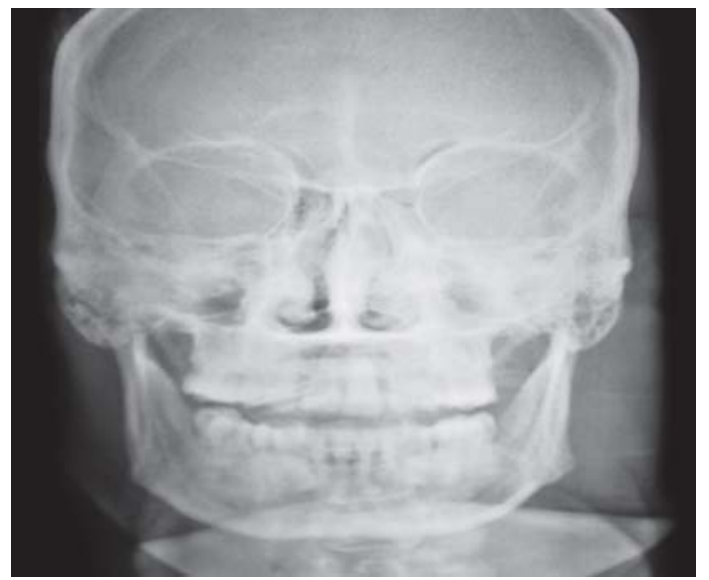

Fig. 2: Posteroanterior view of skull showing fracture of left infraorbital margin and partial obliteration of left maxillary sinus

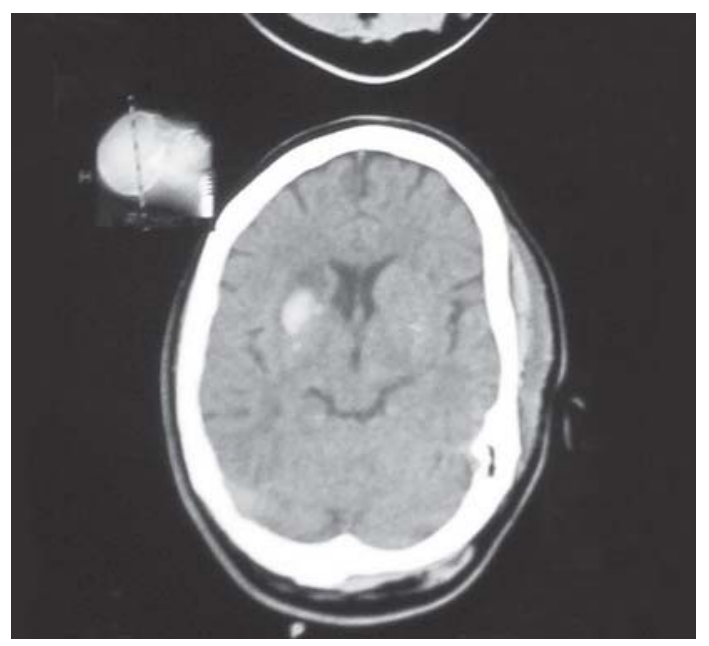

Fig. 3: CT scan of brain showing right basal ganglion bleeding

carried out through conventional method and the specimens were sent for microbiological culture and histopathological examination (Fig. 4). The site was irrigated with $\mathrm{H}_{2} \mathrm{O}_{2}$ and topical gentamicin solution. Pyodine dressing was given at 


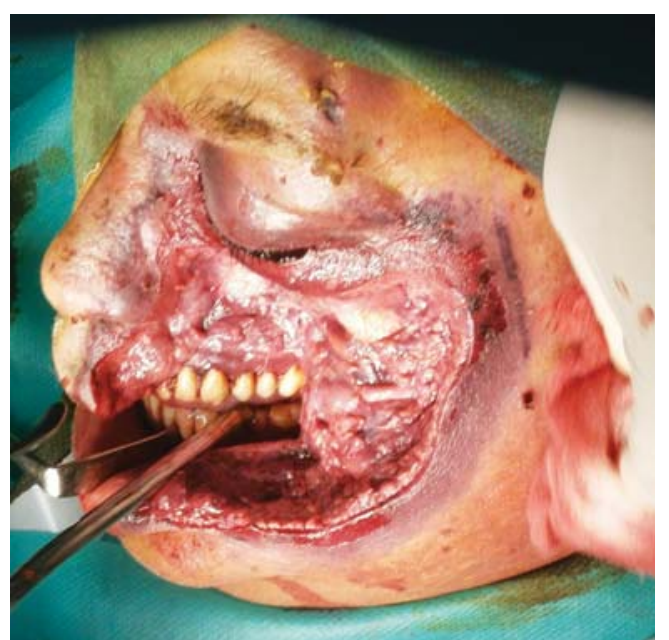

Fig. 4: Clinical picture during debridement showing extensive spread of infection to different maxillofacial structures

the surgical site and the patient was shifted to surgical ICU for postsurgical care.

A CV P line and Foley's catheter were passed while the feeding was carried out through nasogastric tube. For 36 hours patient maintained his vitals in ICU then his general condition started to deteriorate. Patient was shifted to ventilator. Blood pressure and oxygen saturation was not recordable while heart rate increased to 160 beats/min. A fter another couple of hours patient went into asystolic arrest. Dopamine and dobutamine were given intravenously (IV) at the rate of $6 \mathrm{ug} / \mathrm{kg} / \mathrm{min}$ and $2 \mathrm{ug} / \mathrm{kg} / \mathrm{min}$ respectively and cardiopulmonary resuscitation was carried out for 20 minutes but the patient could not be resuscitated.

The reports of the microbial cultures and histopathological examination of debrided tissue were available 3 days after the death of patient. No growth of microorganisms was detected on microbial culture while histopathological examination showed growth of broad aseptate hyphae that was suggestive of mucormycosis.

\section{DISCUSSION}

The common species involved in human mucormycosis include R hizopus, M ucor and A bsidia. It is known to be a disease of immunocompromised patients with a higher incidence in patients suffering from diabetes mellitus while the incidence of mucormycosis in immunocompetent patients is negligible. ${ }^{5-7}$ M oreover, different studies have proven the link between different types of mucormycosis and a variety of risk factors. For example, rhinocerebral type of mucormycosis is more frequently associated with diabetes ketoacidosis while pulmonary mucormycosis is more common in neutropenic patients. ${ }^{4}$ A mong different types of mucormycosis, the rhinocerebral type is most common and this patient was also found to be suffering from a variant of this type. ${ }^{5,8} \mathrm{H}$ yperglycemia with diabetic ketoacidosis predisposed our patient to such opportunistic infections. Rhizopus species are known to have an active ketone reductase system that enables them to breed in acidic environment with high concentration of glucose. ${ }^{9}$

A typical rhinocerebral mucormycosis starts with the inhalation of the spores but invasion to the soft tissue through a breach in the skin or mucosa has also been documented. ${ }^{10}$ The latter is believed to happen with the patient in this case. Once the spores are inside the upper respiratory tract they start growing in nasal cavity and invade the paranasal sinuses. The extension to the intracranial tissues usually happens by direct extension through the superior orbital vein, superior orbital fissure or the cribriform plate. ${ }^{11}$ In the case presented above, the infection was believed to spread from the primary cutaneous lesion to the paranasal sinuses and the orbit. The spread was facilitated by the fractured infraorbital bony margin.

The definite diagnosis of this condition can be made only by growing the fungus in culture media and by detecting characteristic hyphae. ${ }^{4} \mathrm{M}$ ucorales have broad aseptate hyphae that branch out at right angles. CT scan and MRI of brain are of great help in diagnosing intracerebral lesions associated with this disease.

The recommended treatment for mucormycosis includes extensive local debridement accompanied by antifungal therapy with amphotericin B 1 to $1.5 \mathrm{mg} / \mathrm{kg}$ a day. The aggressive surgical management usually results in a large open wound but facial reconstruction with the help of tissue expanders remain a practical option. ${ }^{12}$ The aggressive management of the underlying illness is mandatory to improve the immune status of the patient. Where amphotericin B has proved its effectiveness against Mucorales species, newer under trial drugs of Triazole group, such as posaconazole, also showed antifungal properties both in vitro and in animal models. ${ }^{13,14}$ Tissue hypoxia and acidosis can be reduced to some extent by the use of hyperbaric oxygen therapy but the efficacy of this mode of treatment in such cases is still debatable. ${ }^{5,15}$ In spite of aggressive management of this condition with extensive debridement and administration of drugs with multiple known toxic effects the mortality rate of mucormycosis remains between 30 and $69 \% .{ }^{15} \mathrm{~A}$ disseminated infection of mucormycosis has a fatality rate of over $90 \% .{ }^{16}$

It is recommended that all patients, especially the elderly, should be thoroughly evaluated for the presence of comorbidities and once different factors affecting host defenses are detected, prompt and aggressive management options should be employed. Every nonhealing rapidly progressive lesion should be seen with a high level of suspicion. U rgent histological examination must be carried out in addition to routine microbiological culture and 
sensitivity test to distinguish this condition from necrotizing fasciitis. Since, the incidence of mucormycosis is increasing, medical professionals should revive their knowledge to ensure the timely diagnosis and proper management of this condition.

\section{CONCLUSION}

Mucormycosis is unyielding infectious disease of diabetic patients with a high mortality rate. Undiagnosed immunocompromised states and late or missed diagnosis remain the key issues need to be addressed in developing countries. A high index of clinical suspicion, aggressive diagnostic measures combined with prompt medical and surgical treatment are necessary to improve the outcome.

\section{REFERENCES}

1. Bitar D, V an Cauteren D, Lanternier F, Dannaoui E, Che D, Dromer $F$, et al. Increasing incidence of zygomycosis (mucormycosis), France, 1997-2006. Emerg Infect Dis 2009 Sep;15(9):1395-401.

2. L eitner C, Hoffmann J , Zerfowski M , R einert S. M ucormycosis: Necrotizing soft tissue lesion of the face. J O ral M axillofac Surg 2003 N ov;61(11):1354-58.

3. Prasad RM, B ose SM, V aiphei K, V erma GR. Postoperative abdominal wall mucormycosis mimicking as bacterial necrotising fasciitis. J Postgrad M ed 2003 A pr-J un;49(2): 187-88.

4. A mmari L, K ilani $B$, Tiouiri $H, K$ anoun $F, G$ oubontini $A, M$ nif $E$, et al. M ucormycosis: Four case reports. Tunis M ed 2008 Feb;86(2):165-68.

5. PrabhuRM , Patel R. M ucormycosis and entomophthoromy cosis: A review of the clinical manifestations, diagnosis and treatment. Clin M icrobiol Infect 2004 M ar; 10(Suppl 1):31-47.

6. Buhl MR, Joseph TP, Snelling BE, Buhl L. Temporofacial zygomycosis in a pregnant woman. Infection 1992 J ulAug;20(4):230-32.

7. Pagano L, Ricci P, Tonso A, Nosari A, Cudillo L, M ontillo M, et al. Mucormycosis in patients with haematological malignancies: A retrospective clinical study of 37 cases. GIMEMA Infection Program (Gruppo Italiano Malattie
Ematologiche Maligne dell'A dulto). B J J Haematol 1997 Nov;99(2):331-36.

8. Parfrey NA. Improved diagnosis and prognosis of mucormycosis. A clinicopathologic study of 33 cases. M edicine (B altimore) $1986 \mathrm{M}$ ar;65(2):113-23.

9. Ferguson $B J$. M ucormycosis of the nose and paranasal sinuses. Otolaryngol Clin North A m 2000 A pr;33(2):349-65.

10. Sugar AM. Mucormycosis. Clin Infect Dis 1992 Mar;14(Suppl 1):S126-29.

11. Zapico A DV, Suarez AR, Encinas PM, A ngulo CM, Pozuelo EC. M ucormycosis of the sphenoidal sinus in an otherwise heal thy patient. Case report and literature review. J Laryngol Otol 1996;110:471-73.

12. Tidwell J, Higuera S, Hollier L H J r. Facial reconstruction after mucormycosis in an immunocompetent host. A m J Otolaryngol 2005 Sep-Oct;26(5):333-36

13. Greenberg RN, Scott LJ, V aughn HH, Ribes JA. Zygomycosis (mucormycosis): Emerging clinical importance and new treatments. Curr Opin Infect Dis 2004 D ec;17(6):517-25.

14. K ok J, Gilroy N, Halliday C, Lee OC, Novakovic D, K evin P, et al. Early use of posaconazole in the successful treatment of rhino-orbital mucormycosis caused by R hizopus oryzae. J Infect 2007 Sep;55(3):e33-36.

15. Warwar RE, Bullock JD. Rhino-orbital-cerebral mucormycosis: A review. Orbit $1998 \mathrm{Dec} ; 17(4): 237-45$.

16. Spellberg B, Edwards J J r, Ibrahim A. Novel perspectives on mucormycosis: Pathophysiology, presentation and management. Clin M icrobiol Rev 2005 J ul;18(3):556-69.

\section{ABOUT THE AUTHORS}

\section{Waqar Jeelani (Corresponding Author)}

Dental Surgeon, D epartment of Orthodontics, U niversity M edical and Dental College, Faisalabad, Pakistan

Correspondence Address: P570, Canal W est V iew, A min Town Faisalabad, Pakistan, Phone: 00923457936578, e-mail: wjeelani@yahoo.com

\section{Yousaf Kamal}

House Officer, D epartment of Oral and M axillofacial Surgery, Pakistan Institute of M edical Sciences, Islamabad, Pakistan 